\title{
Pervasive and weakly pervasive pre-Riesz spaces
}

\author{
Anke Kalauch, Helena Malinowski ${ }^{\dagger}$ \\ November 9, 2018
}

\begin{abstract}
Pervasive pre-Riesz spaces are defined by means of vector lattice covers. To avoid the computation of a vector lattice cover, we give two distinct intrinsic characterizations of pervasive pre-Riesz spaces. We introduce weakly pervasive pre-Riesz spaces and observe that this property can be easily checked in examples. We relate weakly pervasive pre-Riesz spaces to pre-Riesz spaces with the Riesz decomposition property.
\end{abstract}

Keywords: Pre-Riesz space, pervasive, Riesz decomposition property Mathematics Subject Classification (2010): 46A40, 06F20

\section{Introduction}

In the analysis of ordered vector spaces which are not vector lattices there are certain additional properties, such as pervasiveness, fordability and the Riesz decomposition property, which allow to generalize well-known results from the vector lattice theory. In the present paper we characterize and relate some of these properties in pre-Riesz spaces.

An ordered vector space $X$ is called pre-Riesz if there is a vector lattice $Y$ and a bipositive linear map $i: X \rightarrow Y$ such that $i(X)$ is order dense in $Y$. The pair $(Y, i)$ is then called a vector lattice cover of $X$. The theory of pre-Riesz spaces and their vector lattice covers is due to van Haandel, see [13]. Pre-Riesz spaces cover a wide range of examples, in particular every Archimedean directed ordered vector space is a pre-Riesz space.

We mainly deal with pervasive pre-Riesz spaces, i.e. spaces $X$ such that for every $y \in Y$ with $y>0$ there is $x \in X$ with $0<i(x) \leqslant y$. Pervasive pre-Riesz spaces were introduced in [7]. To illustrate their importance, we list some results from the literature where pervasive pre-Riesz spaces play an essential role. In [7, Theorem 2.6] pervasiveness is a crucial assumption to show that the restriction of a band in $Y$ to $X$ is a band in $X$. Moreover, it is established that the space $L_{r}\left(\ell_{0}^{\infty}\right)$ of regular

*FR Mathematik, Institut für Analysis, TU Dresden, 01062 Dresden, Germany, anke.kalauch@tu-dresden.de

${ }^{\dagger}$ Unit for BMI, North-West University, Private Bag X6001, Potchefstroom, 2520, South Africa, lenamalinowski@gmx.de 
operators on the space $\ell_{0}^{\infty}$ of eventually constant sequences is a pervasive pre-Riesz space. This yields that the space $L_{o c}\left(\ell_{0}^{\infty}\right)$ of order continuous operators is a band in $L_{r}\left(\ell_{0}^{\infty}\right)$. This statement is a first instance of an Ogasawara type result where the range space is not Dedekind complete. In [4, Example 22] the authors prove that $L_{o c}\left(\ell_{0}^{\infty}\right)$ is pervasive. In [11, Theorem 14] it is shown that in a pervasive preRiesz space with the Riesz decomposition property every directed order closed ideal with a directed double disjoint complement is a band. In [5, Theorem 27] it is established that for a directed ideal $I$ in $X$ a vector lattice cover of $I$ is given by the smallest extension ideal of $I$ in $Y$, provided $X$ is pervasive. In [14, Theorem 5.3] the author obtains that the inverse of a bijective Riesz* homomorphism between pre-Riesz spaces is again a Riesz* homomorphism, provided the pre-image space is pervasive. In [14, Theorem 7.2] spaces of differentiable functions which are defined on sufficiently smooth manifolds and vanish at infinity are shown to be pervasive pre-Riesz spaces.

So far, the definition of pervasiveness is given using a vector lattice cover. In examples it might be difficult to find a convenient representation of a vector lattice cover of a given pre-Riesz space. Therefore in Section 3 we give two intrinsic characterizations of pervasiveness, which do not use vector lattice covers. In Section 4 we introduce weakly pervasive pre-Riesz spaces and give a characterization, such that this property can be easily checked in examples. We relate pervasive and weakly pervasive pre-Riesz spaces to pre-Riesz spaces with the Riesz decomposition property. The following diagram illustrates the results and counterexamples obtained in Section 4.

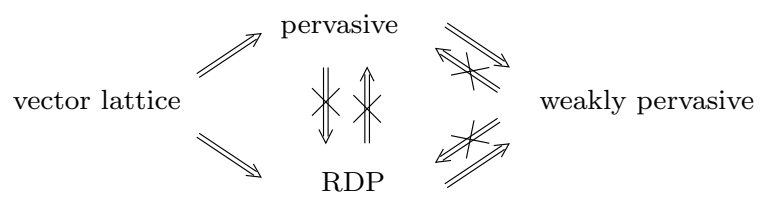

Since we characterize the weak pervasiveness intrinsically (i.e. without using vector lattice covers), our results provide a convenient method to show that a given preRiesz space is not pervasive. This is used to answer the open question whether the space in [12] is pervasive, see Example 14 below.

\section{Preliminaries}

Let $X$ be a real vector space and let $X_{+}$be a cone in $X$, that is, $X_{+}$is a wedge $\left(x, y \in X_{+}\right.$and $\lambda, \mu \geqslant 0$ imply $\left.\lambda x+\mu y \in X_{+}\right)$and $X_{+} \cap\left(-X_{+}\right)=\{0\}$. In $X$ a partial order is defined by $x \leqslant y$ whenever $y-x \in X_{+}$. The space $\left(X, X_{+}\right)$(or, loosely $X$ ) is then called a (partially) ordered vector space.

An ordered vector space $X$ is called Archimedean if for every $x, y \in X$ with $n x \leqslant y$ for every $n \in \mathbb{N}$ one has $x \leqslant 0$. Clearly, every subspace of an Archimedean ordered vector space is Archimedean. The ordered vector space $X$ is called directed if for every $x, y \in X$ there is $z \in X$ such that $x, y \leqslant z$. The space $X$ is directed if and only if $X_{+}$is generating in $X$, that is, $X=X_{+}-X_{+}$. An ordered vector space $X$ has the Riesz decomposition property $(R D P)$ if for every $x_{1}, x_{2}, z \in X_{+}$with $z \leqslant x_{1}+x_{2}$ there exist $z_{1}, z_{2} \in X_{+}$such that $z=z_{1}+z_{2}$ with $z_{1} \leqslant x_{1}$ and $z_{2} \leqslant x_{2}$. The space 
$X$ has the RDP if and only if for every $x_{1}, x_{2}, x_{3}, x_{4} \in X$ with $x_{1}, x_{2} \leqslant x_{3}, x_{4}$ there exists $z \in X$ such that $x_{1}, x_{2} \leqslant z \leqslant x_{3}, x_{4}$. For standard notations in the case that $X$ is a vector lattice see [1].

For two elements $a, b \in X$ of an ordered vector space $X$ we use the notation $] a, b]:=$ $\{x \in X \mid a<x \leqslant b\}$. For a set $A \subseteq X$ we set $A+x:=\{a+x \mid a \in A\}$. Moreover, we write $A \leqslant x$ if and only if for every $a \in A$ we have $a \leqslant x$. By [10, Theorem 13.1] for a set $A \subseteq X$ such that $\sup A$ exists in $X$ and an element $x \in X$ we have that the supremum $\sup (x+A)$ exists in $X$ and

$$
x+\sup A=\sup (x+A) \text {. }
$$

Clearly, we can replace the supremum by the infimum.

We call a linear subspace $D$ of an ordered vector space $X$ order dense in $X$ if for every $x \in X$ we have

$$
x=\inf \{z \in D \mid x \leqslant z\},
$$

see [3, p. 360]. A linear subspace $D$ of $X$ is majorizing in $X$ if for every $x \in X$ there exists $d \in D$ with $x \leqslant d$. For a subset $M \subseteq X$ denote the set of all upper bounds of $M$ by $M^{u}:=\{x \in X \mid \forall m \in M: m \leqslant x\}$. Two elements $x, y \in X$ are called disjoint, in symbols $x \perp y$, if $\{x+y,-x-y\}^{u}=\{x-y,-x+y\}^{u}$, for motivation and details see [6]. For a subset $M \subseteq X$ define $M^{\mathrm{d}}:=\{x \in X \mid \forall m \in M: m \perp x\}$. If $X$ is a vector lattice, then this notion of disjointness coincides with the usual one, see [1, Theorem 1.4(4)]. Let $Y$ be an ordered vector space, $X$ an order dense subspace of $Y$, and $x, y \in X$. Then the disjointness notions in $X$ and $Y$ coincide, i.e. $x \perp y$ in $X$ holds if and only if $x \perp y$ in $Y$, see [6, Proposition 2.1(ii)].

We say that a linear subspace $D$ of a vector lattice $X$ generates $X$ as a vector lattice if for every $x \in X$ there exist finite sets $A, B \subseteq D$ such that $x=\bigvee A-\bigvee B$. Recall that a linear map $i: X \rightarrow Y$, where $X$ and $Y$ are ordered vector spaces, is called bipositive if for every $x \in X$ one has $i(x) \geqslant 0$ if and only if $x \geqslant 0$. An embedding is a bipositive linear map, which implies injectivity. For an ordered vector space $X$, the following statements are equivalent, see [13, Corollaries 4.9-4.11 and Theorems 3.7, 4.13]:

(i) There exist a vector lattice $Y$ and an embedding $i: X \rightarrow Y$ such that $i(X)$ is order dense in $Y$.

(ii) There exist a vector lattice $\tilde{Y}$ and an embedding $i: X \rightarrow \tilde{Y}$ such that $i(X)$ is order dense in $\tilde{Y}$ and generates $\tilde{Y}$ as a vector lattice.

If $X$ satisfies (i), then $X$ is called a pre-Riesz space, and $(Y, i)$ is called a vector lattice cover of $X$. For an intrinsic definition of pre-Riesz spaces see [13]. If $X$ is a subspace of $Y$ and $i$ is the inclusion map, we write briefly $Y$ for $(Y, i)$. As all spaces $\tilde{Y}$ in (ii) are Riesz isomorphic, we call the pair $(\tilde{Y}, i)$ the Riesz completion of $X$ and denote it by $X^{\varrho}$. The space $X^{\varrho}$ is the smallest vector lattice cover of $X$ in the sense that every vector lattice cover $Y$ of $X$ contains a Riesz subspace that is Riesz isomorphic to $X^{\varrho}$. By definition, for every $y \in X^{\varrho}$ there are finite sets $A, B \subseteq X$ such that

$$
y=\bigvee i(A)-\bigvee i(B)
$$


Lemma 1. Let $X$ be a pre-Riesz space and $X^{\varrho}$ its Riesz completion. Let $y \in X^{\varrho}$. Then in (2) the sets $A$ and $B$ can be chosen to be contained in $X_{+}$.

Proof. Let $y \in X^{\varrho}$ have a representation as in $(2)$, i.e. $y=\bigvee i(\tilde{A})-\bigvee i(\tilde{B})$ with $\tilde{A}, \tilde{B} \subseteq X$. Since the pre-Riesz space $X$ is directed there exists $x \in X$ with $0 \leqslant x$, $\tilde{A} \leqslant x$ and $\tilde{B} \leqslant x$. Then

$$
\begin{aligned}
y & =\bigvee i(\tilde{A})-\bigvee i(\tilde{B})=(i(x)-\bigvee i(\tilde{B}))-(i(x)-\bigvee i(\tilde{A}))= \\
& =\bigvee i(x-\tilde{B})-\bigvee i(x-\tilde{A}),
\end{aligned}
$$

where the last equality follows by (1). Clearly, for $A:=x-\tilde{B}$ and $B:=x-\tilde{A}$ we have $A, B \subseteq X_{+}$.

By [13, Theorem 17.1] every Archimedean directed ordered vector space is a preRiesz space. Moreover, every pre-Riesz space is directed. If $X$ is an Archimedean directed ordered vector space, then every vector lattice cover of $X$ is Archimedean. Let $X$ be a pre-Riesz space and $(Y, i)$ a vector lattice cover of $X$. The space $X$ is called pervasive in $Y$ if for every $y \in Y_{+}, y \neq 0$, there exists $x \in X$ such that $0<i(x) \leqslant y$. By [9, Proposition 2.8.8] the space $X$ is pervasive in $Y$ if and only if $X$ is pervasive in any vector lattice cover. Then $X$ is simply called pervasive. The space $X$ is called fordable in $Y$ if for every $y \in Y$ there exists a set $S \subseteq X$ such that $\{y\}^{\mathrm{d}}=i(S)^{\mathrm{d}}$ in $Y$. By $[9$, Proposition 4.1.18] the space $X$ is fordable in $Y$ if and only if $X$ is fordable in any vector lattice cover of $X$. Then $X$ is simply called fordable. By [7, Lemma 2.4] every pervasive pre-Riesz space is fordable.

\section{Intrinsic characterizations of pervasiveness}

In a pre-Riesz space, the definition of pervasiveness depends on the Riesz completion or a vector lattice cover. An intrinsic definition allows to check whether a space is pervasive without having to compute a vector lattice cover. In Theorems 5 and 7 we give two distinct intrinsic characterizations. We start with a recollection of two (non-intrinsic) characterizations of pervasiveness, which we include for the sake of completeness. For the following result from [15, Theorem 4.15, Corollary 4.16] a short proof can also be found in [11, Lemma 1].

Proposition 2. Let $X$ be an Archimedean pre-Riesz space and $(Y, i)$ a vector lattice cover of $X$. Then the following statements are equivalent.

(i) $X$ is pervasive.

(ii) $\forall a \in X \forall y \in Y(i(a)<y \Rightarrow \exists x \in X: i(a)<i(x) \leqslant y)$.

(iii) For every $y \in Y_{+}$with $y \neq 0$ we have $\left.\left.y=\sup (i(X) \cap] 0, y\right]\right)$.

(iv) For every $y \in Y$ and $z \in X$ with $i(z)<y$ we have $y=\sup (i(X) \cap] i(z), y])$.

The following result was established in [5, Theorem 26]. 
Proposition 3. Let $X$ be an Archimedean pre-Riesz space, $Y$ a vector lattice and $i: X \rightarrow Y$ a bipositive linear map. Then the following statements are equivalent.

(i) $i(X)$ is order dense in $Y$ and $X$ is pervasive.

(ii) $i(X)$ is majorizing in $Y$, and for every $y \in Y_{+}$there is $A \subseteq i(X)_{+}$such that $y=\sup A$.

For our first intrinsic characterization of pervasiveness we begin with the following technical statement.

Lemma 4. Let $X$ be a pre-Riesz space and $y \in X^{\varrho}$. Then

(i) $y \geqslant 0$ if and only if for every two finite sets $A, B \subseteq X_{+}$with $y=\bigvee i(A)-$ $\bigvee i(B)$ we have $A^{u} \subseteq B^{u}$.

(ii) $y>0$ if and only if for every two finite sets $A, B \subseteq X_{+}$with $y=\bigvee i(A)-$ $\bigvee i(B)$ we have $A^{u} \subsetneq B^{u}$.

Proof. (i): Let $y \in X^{\varrho}$ with $y \geqslant 0$ and let $A, B \subseteq X_{+}$be finite sets such that $y=\bigvee i(A)-\bigvee i(B)$. The existence of such sets is guaranteed by Lemma 1 . Then we have $\bigvee i(B) \leqslant \bigvee i(A)$. If $v \in X^{\varrho}$ is an upper bound of $i(A)$, then it is an upper bound of $i(B)$, i.e. $i(A)^{u} \subseteq i(B)^{u}$. It follows $A^{u}=\left[i(A)^{u}\right] i \subseteq\left[i(B)^{u}\right] i=B^{u}$.

Let, on the other hand, $y \in X^{\varrho}$ and let $A, B \subseteq X_{+}$be finite sets with $y=\bigvee i(A)-$ $\bigvee i(B)$ and $A^{u} \subseteq B^{u}$. We show $i(A)^{u} \subseteq i(B)^{u}$. Let $v \in i(A)^{u}$. Due to $X$ being order dense in $X^{\varrho}$, for the set $V:=\{i(x) \mid x \in X, v \leqslant i(x)\} \subseteq i(X)$ we have $v=\inf V$ in $X^{\varrho}$. For every $x \in X$ with $i(x) \in V$ from $i(A) \leqslant v \leqslant i(x)$ we obtain $A \leqslant x$, i.e. $x \in A^{u} \subseteq B^{u}$. It follows $i(x) \in i(B)^{u}$, i.e. $i(B) \leqslant i(x)$. As this is true for every $x \in X$ with $i(x) \in V$, it follows $i(B) \leqslant \inf V=v$. That is, $v \in i(B)^{u}$. We obtain $i(A)^{u} \subseteq i(B)^{u}$, from which we conclude $\bigvee i(B) \leqslant \bigvee i(A)$ and thus $y \geqslant 0$.

(ii): Let $y \in X^{\varrho}$ with $y>0$ and let $A, B \subseteq X_{+}$be finite sets such that $y=$ $\bigvee i(A)-\bigvee i(B)$. Then $y \geqslant 0$, thus (i) implies $A^{u}=B^{u}$. We show $A^{u} \neq B^{u}$ by contradiction. Assume, on the contrary, that $A^{u}=B^{u}$. Due to $y>0$ we have $t:=\bigvee i(B)<\bigvee i(A)=: s$. Since $X$ is order dense in $X^{\varrho}$ it follows

$$
\begin{aligned}
t & =\inf \{i(x) \mid x \in X, t \leqslant i(x)\}=\inf \{i(x) \mid x \in X, A \leqslant x\}=\inf i\left(A^{u}\right)= \\
& =\inf i\left(B^{u}\right)=\inf \{i(x) \mid x \in X, s \leqslant i(x)\}=s,
\end{aligned}
$$

a contradiction. We conclude that $A^{u} \subsetneq B^{u}$.

Let, on the other hand, $y \in X^{\varrho}$ and let $A, B \subseteq X_{+}$be finite sets with $y=\bigvee i(A)-$ $\bigvee i(B)$ and $A^{u} \subsetneq B^{u}$. Then $A^{u} \subseteq B^{u}$ and (i) imply $y \geqslant 0$. We show $y>0$ by contradiction. Let, on the contrary, $y=0$. Then $t:=\bigvee i(B)=\bigvee i(A)=: s$. For a fixed $x \in X$ the statement $A \leqslant x$ is equivalent to $\bigvee i(A) \leqslant i(x)$, and similarly, $B \leqslant x$ is equivalent to $\bigvee i(B) \leqslant i(x)$. It follows

$$
\begin{aligned}
A^{u} & =\{x \in X \mid A \leqslant x\}=\{x \in X \mid t \leqslant i(x)\}=\{x \in X \mid s \leqslant i(x)\}= \\
& =\{x \in X \mid B \leqslant x\}=B^{u},
\end{aligned}
$$

a contradiction to $A^{u} \subsetneq B^{u}$. We conclude that $y>0$. 
Theorem 5. Let $X$ be a pre-Riesz space. Then $X$ is pervasive if and only if for every two finite sets $A, B \subseteq X_{+}$with $A^{u} \subsetneq B^{u}$ there exists $x \in X, x>0$, such that $A^{u} \subseteq(x+B)^{u}$.

Proof. " $\Rightarrow$ ": Let $X$ be pervasive and let $\left(X^{\varrho}, i\right)$ be the Riesz completion of $X$. Let $A, B \subseteq X_{+}$be finite sets with $A^{u} \subsetneq B^{u}$. Set $y:=\bigvee i(A)-\bigvee i(B)$. Then by Lemma 4(ii) it follows $y>0$. As $X$ is pervasive, there exists an $x \in X$ with $0<i(x) \leqslant y$. Hence

$$
0 \leqslant y-i(x)=\bigvee i(A)-(\bigvee i(B)+i(x))=\bigvee i(A)-(\bigvee i(B+x))
$$

Clearly, $B+x \subseteq X_{+}$, therefore by Lemma 4(i) we obtain $A^{u} \subseteq(x+B)^{u}$.

" $\Leftarrow$ ": Let for every two finite sets $A, B \subseteq X_{+}$with $A^{u} \subsetneq B^{u}$ there exist $x \in X_{+}$, $x \neq 0$, such that $A^{u} \subseteq(x+B)^{u}$. Let $y \in X^{\varrho}$ with $y>0$. Then by Lemma 1 there exist finite sets $A, B \subseteq X_{+}$such that $y$ has the representation $y=\bigvee i(A)-\bigvee i(B)$. As $y>0$, by Lemma 4(ii) it follows $A^{u} \subsetneq B^{u}$. By assumption there exists $x \in X$, $x>0$, such that $A^{u} \subseteq(x+B)^{u}$. Lemma 4 (i) yields $0 \leqslant y-i(x)$, i.e. $X$ is pervasive.

Next we intend to give a characterization of pervasiveness using pairs of elements instead of finite subsets.

Proposition 6. Let $X$ be a pre-Riesz space and $(Y, i)$ a vector lattice cover of $X$. Then the following are equivalent.

(i) $X$ is pervasive.

(ii) For every $b_{1}, b_{2} \in X$ with $i\left(b_{1}\right) \vee i\left(b_{2}\right)>0$ there exists $x \in X$ such that $0<i(x) \leqslant i\left(b_{1}\right) \vee i\left(b_{2}\right)$

(iii) For every $b \in X$ with $i(b) \vee 0>0$ there exists $x \in X$ such that $0<i(x) \leqslant$ $i(b) \vee 0$.

Proof. The implications (i) $\Rightarrow$ (ii) and (ii) $\Rightarrow$ (iii) are clear.

(iii) $\Rightarrow($ i): Let $y \in Y$ with $y>0$. Since $X$ is order dense in $Y$, we have $y=$ $\sup \{z \in i(X) \mid z \leqslant y\}$. Thus there exists a lower bound $z \in i(X)$ of $y$ for which we have $z \notin 0$. For $b:=i^{-1}(z)$ it follows $0<i(b) \vee 0$. By assumption there exists $x \in X$ such that $0<i(x) \leqslant i(b) \vee 0$. It follows $0<i(x) \leqslant i(b) \vee 0 \leqslant y$, i.e. $X$ is pervasive.

By reformulating Proposition 6 with the help of upper bounds we obtain the following intrinsic characterization of pervasiveness. This characterization is used in Example 13 below, where we establish that a pre-Riesz space is pervasive without prior computation of its vector lattice cover.

Theorem 7. Let $X$ be an Archimedean pre-Riesz space. Then the following are equivalent.

(i) $X$ is pervasive.

(ii) For every $b_{1}, b_{2} \in X$ with $\left\{b_{1}, b_{2}\right\}^{u} \subseteq X_{+} \backslash\{0\}$ there is $x \in X$ such that for every $u \in\left\{b_{1}, b_{2}\right\}^{u}$ we have $0<x \leqslant u$. 
(iii) For every $b \in X$ with $b \notin 0$ there is $x \in X$ such that for every $u \in X_{+}$the inequality $b \leqslant u$ implies $0<x \leqslant u$.

Proof. Let $(Y, i)$ be a vector lattice cover of $X$.

(i) $\Rightarrow$ (ii): Let $b_{1}, b_{2} \in X$ be two elements such that $0<u^{\prime}$ holds for every upper bound $u^{\prime} \in\left\{b_{1}, b_{2}\right\}^{u}$. Since $X$ is order dense in $Y$, this implies

$$
\begin{aligned}
i\left(b_{1}\right) \vee i\left(b_{2}\right) & =\inf \left\{z \in i(X) \mid i\left(b_{1}\right) \vee i\left(b_{2}\right) \leqslant z\right\}= \\
& =\inf \left\{i\left(u^{\prime}\right) \mid u^{\prime} \in\left\{b_{1}, b_{2}\right\}^{u}\right\} \geqslant 0 .
\end{aligned}
$$

Now, if $i\left(b_{1}\right) \vee i\left(b_{2}\right)=0$, then the element 0 is an upper bound of $b_{1}, b_{2}$. This is a contradiction to the assumption that $u^{\prime}>0$ for every upper bound $u^{\prime} \in\left\{b_{1}, b_{2}\right\}^{u}$. Therefore we have $i\left(b_{1}\right) \vee i\left(b_{2}\right)>0$. Since $X$ is pervasive, there exists $x \in X$ with $0<i(x) \leqslant i\left(b_{1}\right) \vee i\left(b_{2}\right)$. This implies $0<x \leqslant u$ for every upper bound $u \in\left\{b_{1}, b_{2}\right\}^{u}$. (ii) $\Rightarrow\left(\right.$ i): We use the characterization in Proposition 6(ii). Let $b_{1}, b_{2} \in X$ be such that $i\left(b_{1}\right) \vee i\left(b_{2}\right)>0$. We have to show that there exists an element $x \in X$ with $0<i(x) \leqslant i\left(b_{1}\right) \vee i\left(b_{2}\right)$. Let $u^{\prime} \in\left\{b_{1}, b_{2}\right\}^{u}$. Then $b_{1}, b_{2} \leqslant u^{\prime}$ yields $0<i\left(b_{1}\right) \vee i\left(b_{2}\right) \leqslant$ $i\left(u^{\prime}\right)$, which implies $0<u^{\prime}$. By assumption there exists $x \in X$ such that for every $u \in\left\{b_{1}, b_{2}\right\}^{u}$ we have $0<x \leqslant u$. Due to $X$ being order dense in $Y$ we obtain in $Y$ the inequality

$$
0<i(x) \leqslant \inf \left\{i(u) \mid u \in X, i\left(b_{1}\right) \vee i\left(b_{2}\right) \leqslant i(u)\right\}=i\left(b_{1}\right) \vee i\left(b_{2}\right) .
$$

The characterization in Proposition 6(ii) implies that $X$ is pervasive.

(i) $\Rightarrow$ (iii): Let $b \in X$ with $b \notin 0$. We show

$$
\exists x \in X \forall u \in X_{+}: \quad b \leqslant u \Rightarrow 0<x \leqslant u \text {. }
$$

We have $i(b) \vee 0 \geqslant 0$. If $i(b) \vee 0=0$, then $b \leqslant 0$, a contradiction. We conclude that $i(b) \vee 0>0$. Since $X$ is pervasive, we obtain that there exists an $x \in X$ with $0<i(x) \leqslant i(b) \vee 0$. That is, for every $u \in X$ with $0, b \leqslant u$ we have $0<i(x) \leqslant$ $i(b) \vee 0 \leqslant i(u)$. This leads to the inequality $0<x \leqslant u$.

(iii) $\Rightarrow(\mathrm{i})$ : We use the characterization in Proposition 6(iii). Let $b \in X$ be such that $i(b) \vee 0>0$. We have to show that there exists $x \in X$ with $0<i(x) \leqslant i(b) \vee 0$.

If $b<0$, then $i(b) \vee 0 \leqslant 0$, a contradiction. We conclude $b \nless 0$. By assumption there exists $x \in X$ such that for every $u \in X_{+}$with $b \leqslant u$ we have $0<x \leqslant u$. Due to $X$ being order dense in $Y$ there exists the infimum in the following inequality:

$$
\begin{aligned}
0 & <i(x) \leqslant \inf \left\{i(u) \mid u \in X_{+} \text {and } b \leqslant u\right\}= \\
& =\inf \{i(u) \mid u \in X \text { and } 0 \vee i(b) \leqslant i(u)\}=0 \vee i(b) .
\end{aligned}
$$

The characterization in Proposition 6(iii) implies that $X$ is pervasive.

\section{Weakly pervasive pre-Riesz spaces}

In this section we relate pervasiveness to other properties of pre-Riesz spaces. In Proposition 6(ii) pervasiveness of a pre-Riesz space $X$ is characterized with the aid of the supremum of two elements of $i(X)$. The question arises whether the supremum can be replaced by the infimum, which yields the following definition. 
Definition 8. Let $X$ be a pre-Riesz space and $(Y, i)$ a vector lattice cover of $X$. Then $X$ is called weakly pervasive (in $Y$ ) if for every $b_{1}, b_{2} \in X$ with $i\left(b_{1}\right) \wedge i\left(b_{2}\right)>$ 0 there exists $x \in X$ such that $0<i(x) \leqslant i\left(b_{1}\right) \wedge i\left(b_{2}\right)$.

It is clear that every pervasive pre-Riesz space is weakly pervasive. The converse is not true, see Example 10 below. We first characterize weakly pervasive pre-Riesz spaces.

Lemma 9. Let $X$ be a pre-Riesz space and $(Y, i)$ a vector lattice cover of $X$. Then the following statements are equivalent.

(i) $X$ is weakly pervasive in $Y$.

(ii) For every $a, b_{1}, b_{2} \in X$ with $i\left(b_{1}\right) \wedge i\left(b_{2}\right)>i(a)$ there exists an element $x \in X$ such that $a<x \leqslant b_{1}, b_{2}$.

(iii) For every $b_{1}, b_{2} \in X$ with $b_{1}, b_{2}>0$ and $b_{1} \not \perp b_{2}$ there exists an element $x \in X$ such that $0<x \leqslant b_{1}, b_{2}$.

Proof. The equivalence (i) $\Leftrightarrow$ (ii) and the implication (i) $\Rightarrow$ (iii) are immediate. To show the implication (iii) $\Rightarrow(\mathrm{i})$, let $b_{1}, b_{2} \in X$ with $i\left(b_{1}\right) \wedge i\left(b_{2}\right)>0$. Then it follows $b_{1}, b_{2}>0$ and $b_{1} \not \subset b_{2}$. By assumption there exists $x \in X$ with $0<x \leqslant b_{1}, b_{2}$, i.e. we have $0 \leqslant i(x) \leqslant i\left(b_{1}\right) \wedge i\left(b_{2}\right)$.

Note that the statement in Lemma 9(iii) does not depend on the vector lattice cover. Similarly to pervasiveness, $X$ is weakly pervasive in $X^{\varrho}$ if and only if $X$ is weakly pervasive in any vector lattice cover of $X$. This justifies that we simply call $X$ weakly pervasive.

As every pervasive pre-Riesz space is fordable, the next example establishes that a weakly pervasive pre-Riesz space need not be pervasive.

Example 10. An Archimedean weakly pervasive pre-Riesz space need not be fordable.

Consider the vector space

$$
Y=\left\{\left(y_{i}\right)_{i \in \mathbb{Z}} \in \ell^{\infty}(\mathbb{Z}) \mid \lim _{i \rightarrow \infty} y_{i} \text { exists }\right\} .
$$

Endowed with the coordinatewise order, $Y$ is an Archimedean vector lattice. In $[8$, Example 5.2] it is shown that the Archimedean directed subspace

$$
X:=\left\{\left(x_{i}\right)_{i \in \mathbb{Z}} \in \ell^{\infty}(\mathbb{Z}) \mid \sum_{k=1}^{\infty} \frac{x_{-k}}{2^{k}}=\lim _{i \rightarrow \infty} x_{i}\right\}
$$

of $Y$ is order dense in $Y$, that is, $X$ is an Archimedean pre-Riesz space and $Y$ is a vector lattice cover of $X$.

We show by contradiction that $X$ is not fordable. Let $z \in \ell^{\infty}(\mathbb{Z})$ be such that $z_{-1}=1$ and $z_{k}=0$ for every $k \neq-1$, i.e. $z=\left(\ldots, 0,0,0,1, z_{0}=0,0,0, \ldots\right) \in Y$. Then we have

$$
\{z\}^{\mathrm{d}}=\left\{y=\left(y_{k}\right)_{k \in \mathbb{Z}} \in Y \mid y_{-1}=0\right\} .
$$


Assume that $X$ is fordable and let $S \subseteq i(X)$ be such that $S^{\mathrm{d}}=\{z\}^{\mathrm{d}}$. Let $x \in Y$ be defined by $x_{-1}=0$ and $x_{k}=1$ for $k \neq-1$, that is, $x=\left(\ldots, 1,1,0, x_{0}=\right.$ $1,1, \ldots)$. Then $x \in\{z\}^{\mathrm{d}}=S^{\mathrm{d}}$. Since for every $s \in S$ we have $s \perp x$, it follows $s=\left(\ldots, 0,0, s_{-1}, s_{0}=0,0,0, \ldots\right)$, where $s_{-1} \in \mathbb{R}$. Clearly, $\lim _{k \rightarrow \infty} s_{k}=0$. Since $s$ belongs to $i(X)$, we obtain

$$
0=\lim _{k \rightarrow \infty} s_{k}=\sum_{k=1}^{\infty} \frac{s_{-k}}{2^{k}}=\frac{s_{-1}}{2} .
$$

This leads to $s_{-1}=0$ and therefore $s=0$. It follows $S=\{0\}$. This implies $S^{\mathrm{d}}=Y \neq\{z\}^{\mathrm{d}}$, a contradiction. We conclude that the pre-Riesz space $X$ is not fordable.

It is left to show that $X$ is weakly pervasive. Let $b, c \in X_{+}$be two non-zero elements with $b \not \subset c$. Due to $i(b) \wedge i(c) \neq 0$ and $b, c>0$ there exists a coordinate $j$ such that $b_{j}, c_{j}>0$. Consider the following two cases.

For the first case, let $j \geqslant 0$. Define the sequence $x=\left(x_{k}\right)_{k \in \mathbb{Z}}$ by $x_{j}:=b_{j} \wedge c_{j}>0$ and $x_{k}:=0$ for $k \neq j$. Then we have $\sum_{k=1}^{\infty} \frac{x_{-k}}{2^{k}}=0=\lim _{k \rightarrow \infty} x_{k}$ and thus $x \in X$ and $0<x \leqslant b, c$.

For the second case, let $j<0$. Set $C:=b_{j} \wedge c_{j}>0$. Since all coordinates of $b$ and $c$ are non-negative, we obtain the following estimates:

$$
\sum_{k=1}^{\infty} \frac{b_{-k}}{2^{k}} \geqslant \frac{b_{j}}{2^{-j}} \geqslant \frac{C}{2^{-j}}>0 \text { and } \quad \sum_{k=1}^{\infty} \frac{c_{-k}}{2^{k}} \geqslant \frac{c_{j}}{2^{-j}} \geqslant \frac{C}{2^{-j}}>0 .
$$

Due to $b, c \in X$ this leads to $\lim _{k \rightarrow \infty} b_{k} \geqslant \frac{C}{2^{-j}}>0$ and $\lim _{k \rightarrow \infty} c_{k} \geqslant \frac{C}{2^{-j}}>0$. Therefore there exist $N \in \mathbb{N}_{0}$ and $\varepsilon \in \mathbb{R}_{>0}$ such that

$$
\text { for every } k>N \text { we have } b_{k}, c_{k} \geqslant \varepsilon \text {. }
$$

Let $\alpha_{1}, \alpha_{2} \in \mathbb{R}$ be defined by $\alpha_{1}:=\min \{\varepsilon, C\}$ and $\alpha_{2}:=\frac{\alpha_{1}}{2^{-j}}$. Due to $j<0$ we obtain $\alpha_{2}=\frac{\min \{\varepsilon, C\}}{2^{-j}} \leqslant \frac{\varepsilon}{2^{-j}}<\varepsilon$. Define a sequence $x=\left(x_{k}\right)_{k \in \mathbb{Z}}$ as follows:

$$
x_{k}:= \begin{cases}\alpha_{1} & \text { for } k=j \\ \alpha_{2} & \text { for } k>N \\ 0 & \text { otherwise }\end{cases}
$$

Then $\lim _{k \rightarrow \infty} x_{k}=\alpha_{2}<\varepsilon$ and $\sum_{k=1}^{\infty} \frac{x_{-k}}{2^{k}}=\frac{\alpha_{1}}{2^{-j}}=\alpha_{2}$, which yields $x \in X$. On the one hand, from $\varepsilon, C>0$ it follows $\alpha_{1}, \alpha_{2}>0$ and therefore $x>0$. On the other hand, we have $x \leqslant b, c$. Indeed, for $k=j$ we obtain

$$
x_{j}=\alpha_{1}=\min \{\varepsilon, C\} \leqslant C=b_{j} \wedge c_{j}
$$

and for $k>N$, using (3) in the last step of the following equation, we have

$$
x_{k}=\alpha_{2}=\min \left\{\frac{\varepsilon}{2^{-j}}, \frac{C}{2^{-j}}\right\} \leqslant \varepsilon \leqslant b_{k}, c_{k} .
$$

We conclude that $0<x \leqslant b, c$, i.e. $X$ is weakly pervasive. 
It remains an open question whether every fordable pre-Riesz space is weakly pervasive $^{1}$. Another important property of pre-Riesz spaces is the RDP, which we next relate to the weak pervasiveness.

Proposition 11. If a pre-Riesz space $X$ has the $R D P$, then $X$ is weakly pervasive.

Proof. Let $X$ be a pre-Riesz space with RDP. We use Lemma 9(iii). Let $b_{1}, b_{2} \in X$ be such that $b_{1}, b_{2}>0$ and $b_{1} \not \perp b_{2}$, i.e. $i\left(b_{1}\right) \wedge i\left(b_{2}\right)>0$ in a vector lattice cover $(Y, i)$ of $X$. We set $a_{1}:=0$. Since $X$ is order dense in $Y$, for the set

$$
S:=\left\{z \in i(X) \mid z \leqslant i\left(b_{1}\right) \wedge i\left(b_{2}\right)\right\}
$$

we have $i\left(b_{1}\right) \wedge i\left(b_{2}\right)=\sup S$. The element $a_{1}=0$ is a lower bound of $i\left(b_{1}\right) \wedge i\left(b_{2}\right)$. Assume that $S \leqslant 0$, then $\sup S \leqslant 0$ leads to a contradiction. Therefore there exists $a_{2} \in X$ with $a_{2} \nless 0$ and such that $i\left(a_{2}\right) \in S$. For the four elements we have the relationship $a_{1}, a_{2} \leqslant b_{1}, b_{2}$. Since $X$ has the RDP, there exists $x \in X$ such that $a_{1}, a_{2} \leqslant x \leqslant b_{1}, b_{2}$. Since $a_{1}=0$ and $a_{2} \nless 0$, it follows $x>0$. We conclude that there exists $x>0$ such that $x \leqslant b_{1}, b_{2}$, i.e. $X$ is weakly pervasive.

Example 12. An Archimedean weakly pervasive pre-Riesz space need not have the $R D P$.

In [11, Example 23] the following pre-Riesz space is considered:

$$
X:=\mathrm{PA}[-1,1] \oplus\{\lambda q \mid \lambda \in \mathbb{R}\}=\{f+\lambda q \mid f \in \mathrm{PA}[-1,1], \lambda \in \mathbb{R}\}
$$

where $q \in C[-1,1], q(t)=t^{2}$ for $t \in[-1,1]$, and $\mathrm{PA}[-1,1]$ is the set of all continuous piecewise affine functions on $[-1,1]$. It is established that $C[-1,1]$ is a vector lattice cover of $X$ with the identity $i$ as the embedding map, and that $X$ is pervasive. Hence weakly pervasive. Moreover, $X$ does not have the RDP.

Remark. It is not clear whether weak pervasiveness is equivalent to the following property:

(P) For every $b_{1}, \ldots, b_{n} \in X$ with $b_{1}, \ldots, b_{n}>0$ and $i\left(b_{1}\right) \wedge \ldots \wedge i\left(b_{n}\right) \neq 0$ there exists an element $x \in X$ such that $0<x \leqslant b_{1}, \ldots, b_{n}$.

However, due to [2, Lemma 1.53] it is straightforward that RDP implies (P). On the other hand, the property $(\mathrm{P})$ does not imply RDP. Indeed, in Example 12 the space $X$ satisfies $(\mathrm{P})$. Let $b_{1}, \ldots, b_{n} \in X$ with $b_{1}, \ldots, b_{n}>0$ be such that $0<$ $i\left(b_{1}\right) \wedge \ldots \wedge i\left(b_{n}\right) \in C[-1,1]$. Then there is a piecewise affine function $y \in C[-1,1]$ such that $0<y \leqslant i\left(b_{1}\right) \wedge \ldots \wedge i\left(b_{n}\right)$. Since $y$ is piecewise affine, we have for $x:=i(y)^{-1} \in X$ that $0<x \leqslant b_{1}, \ldots, b_{n}$. That is, $X$ satisfies $(\mathrm{P})$.

Note that a pervasive pre-Riesz space which does not have the RDP is given in [11, Example 23]. The next example shows that RDP does not imply pervasiveness, in general.

\footnotetext{
${ }^{1}$ In [9, Example 4.1.19] the authors considers a pre-Riesz space $X$ which is fordable, but not pervasive. One can show that $X$ is weakly pervasive.
} 
Example 13. An Archimedean pre-Riesz space with RDP need not be pervasive. Consider the vector space of all (continuous) rational functions on $[0,1]$, i.e.

$$
X:=\left\{x \in C[0,1] \mid x=\frac{p}{q}, \forall t \in[0,1]: q(t) \neq 0, \text { and } p, q \text { polynomial }\right\},
$$

endowed with the pointwise order. Then $X$ is a subspace of the Archimedean vector lattice $C[0,1]$ and hence is Archimedean as well. Clearly, $X$ is directed and therefore pre-Riesz. Riesz established that $X$ has the RDP, see [2, Example 1.56].

To show that $X$ is not pervasive, we use the characterization of Theorem 7 (iii), which avoids the computation of a vector lattice cover of $X$. Let $b(t):=-16\left(t-\frac{1}{4}\right)^{2}+1$ for $t \in[0,1]$. Then the polynomial $b$ belongs to $X$. We have to show that there does not exist $x \in X$ such that $0<x \leqslant u$ holds for every $u \in X$ with $0, b \leqslant u$.

First consider for every $\left.s \in] \frac{1}{2}, 1\right]$ the positive parabola

$$
u_{s}(t):=\frac{1}{\left(\frac{1}{2}-s\right)^{2}}(t-s)^{2} .
$$

Notice that the maximal value of the parabola $b$ is the real number $b\left(\frac{1}{4}\right)=1$, and for $t \in\left[\frac{1}{2}, 1\right]$ we have $b(t) \leqslant 0$. Moreover, for each $u_{s}$ with $\left.\left.s \in\right] \frac{1}{2}, 1\right]$ we obtain $u_{s}\left(\frac{1}{2}\right)=1$ and $u_{s}(s)=0$. It follows for the positive function $u_{s}$ that $b \leqslant u_{s}$ for every $\left.\left.s \in\right] \frac{1}{2}, 1\right]$. Assume now that there exists $x \in X$ such that $0<x \leqslant u$ holds for every positive $u$ with $b \leqslant u$. In particular, we have $0 \leqslant x(s) \leqslant u_{s}(s)=0$ for each $\left.\left.s \in\right] \frac{1}{2}, 1\right]$. That is, $\left.\left.x(] \frac{1}{2}, 1\right]\right)=0$. However, the element $x$ has a representation $x=\frac{p}{q}$ with polynomials $p$ and $q$. Therefore the numerator $p$ must be zero on the whole of the interval $\left.] \frac{1}{2}, 1\right]$. Due to $p$ being polynomial it follows $p=0$, which implies $x=0$, a contradiction to $x>0$. Theorem 7 yields that $X$ is not pervasive.

There are Archimedean pre-Riesz spaces, which are not vector lattices, but are weakly pervasive. A simple example is the space $C^{1}[0,1]$ of differentiable functions on the interval $[0,1]$. On the other hand, not every Archimedean pre-Riesz space is weakly pervasive, as we see in the following example. We use an example from [12], where the focus is to construct an order bounded, non-regular linear functional on an Archimedean directed ordered vector space. The underlying space is a pre-Riesz space, but there is no obvious candidate for a vector lattice cover. So far, it was an open question whether this pre-Riesz space is pervasive. Using the characterization in Lemma 9, we establish that the space is not even weakly pervasive.

Example 14. An Archimedean pre-Riesz space need not be weakly pervasive. As in [12], for $A \subseteq\left[0, \infty\right.$ [ let $\mathbb{1}_{A}$ denote the corresponding indicator function, for $n, k \in \mathbb{N}$ define

$$
\begin{aligned}
e_{n}:\left[0, \infty\left[\rightarrow \mathbb{R}, \quad t \mapsto \mathbb{1}_{[n-1, n[}(t),\right.\right. \\
u_{n, k}:\left[0, \infty\left[\rightarrow \mathbb{R}, \quad t \mapsto n t \mathbb{1}_{\left[0, \frac{1}{n}\right]}(t)+\frac{1}{k} \mathbb{1}_{\left\{n+\frac{1}{k}\right\}}(t),\right.\right.
\end{aligned}
$$

and consider the subspace $X:=\operatorname{span}\left\{e_{n}, u_{n, k} \mid n, k \in \mathbb{N}\right\}$ of $\mathbb{R}^{[0, \infty[}$ with point-wise order. As is established in [12], $X$ is directed. Moreover, since $X$ is a subspace of the Archimedean vector lattice $\mathbb{R}^{[0, \infty[}, X$ is Archimedean and therefore a pre-Riesz space. 
We show that $X$ is not weakly pervasive. To that end we use the characterization in Lemma 9(iii). For $b_{1}:=2 u_{1,2}$ and $b_{2}:=e_{2}$ we have $b_{1}, b_{2}>0$. We show $b_{1} \not \subset b_{2}$ by establishing that $\left\{b_{1}-b_{2},-b_{1}+b_{2}\right\}^{u} \neq\left\{b_{1}+b_{2},-b_{1}-b_{2}\right\}^{u}$. Consider the element $v:=2 u_{1,3}+e_{2}$. We show that $v \in\left\{b_{1}-b_{2},-b_{1}+b_{2}\right\}^{u}$. Indeed, for $t \in[0,1]$ we have $v(t)=2 u_{1,3}(t)=2 t \geqslant \pm 2 t= \pm\left(b_{1}-b_{2}\right)(t)$. For $t \in[1,2] \backslash\left\{\frac{3}{2}, \frac{4}{3}\right\}$ we obtain $v(t)=$ $e_{2}(t)=1 \geqslant \pm 1= \pm\left(b_{1}-b_{2}\right)(t)$. Moreover, we get $v\left(\frac{4}{3}\right)=\frac{5}{3} \geqslant \pm 1= \pm\left(b_{1}-b_{2}\right)\left(\frac{4}{3}\right)$ and $v\left(\frac{3}{2}\right)=1 \geqslant \mp \frac{1}{2}= \pm\left(b_{1}-b_{2}\right)\left(\frac{3}{2}\right)$. We conclude $v \in\left\{b_{1}-b_{2},-b_{1}+b_{2}\right\}^{u}$. On the other hand, $v \notin\left\{b_{1}+b_{2},-b_{1}-b_{2}\right\}^{u}$, as $v\left(\frac{3}{2}\right)=1<2=\left(b_{1}+b_{2}\right)\left(\frac{3}{2}\right)$. This establishes $b_{1} \not \perp b_{2}$.

Assume there is $x \in X$ with $0<x \leqslant b_{1}, b_{2}$. Then for $t \in\left[0, \infty\left[\backslash\left\{\frac{3}{2}\right\}\right.\right.$ we have $x(t)=0$ and $0<x\left(\frac{3}{2}\right) \leqslant 1$. It follows that $x$ is a non-zero multiple of $u_{1,2}$, which is a contradiction. That is, $X$ is not weakly pervasive.

\section{References}

[1] C. D. Aliprantis and O. Burkinshaw. Positive Operators. Springer, New York, 2006.

[2] C. D. Aliprantis and R. Tourky. Cones and Duality, volume 84 of Graduate Studies in Mathematics. American Mathematical Society, Providence, Rhode Island, 2007.

[3] G. Buskes and A.C.M. van Rooij. The vector lattice cover of certain partially ordered groups. J. Austral. Math. Soc. (Series A), 54:352-367, 1993.

[4] A. Kalauch and H. Malinowski. Order continuous operators on pre-Riesz spaces and embeddings. https://arxiv.org/abs/1802.02476, 2018. Submitted.

[5] A. Kalauch and H. Malinowski. Vector lattice covers of ideals and bands in pre-Riesz spaces. Quaestiones Mathematicae, 2018. To appear. https://doi . org/10.2989/16073606.2018.1501620.

[6] A. Kalauch and O. van Gaans. Disjointness in Partially Ordered Vector Spaces. Positivity, 10(3):573-589, 2006.

[7] A. Kalauch and O. van Gaans. Bands in Pervasive pre-Riesz Spaces. Operators and Matrices, 2(2):177-191, 2008.

[8] A. Kalauch and O. van Gaans. Ideals and Bands in Pre-Riesz Spaces. Positivity, 12(4):591-611, 2008.

[9] A. Kalauch and O. van Gaans. Pre-Riesz spaces. De Gruyter, Berlin, 2018.

[10] W. A. J. Luxemburg and A. C. Zaanen. Riesz Spaces I. North-Holland Publ. Comp., Amsterdam, 1971.

[11] H. Malinowski. Order closed ideals in pre-Riesz spaces and their relationship to bands. Positivity, 22(4):1039-1063, 2018. https://arxiv.org/abs/1801. 09963. 
[12] O. van Gaans. An elementary example of an order bounded dual space that is not directed. Positivity, 9(2):265-267, 2005.

[13] M. van Haandel. Completions in Riesz Space Theory. Proefschrift (PhD thesis), Katholieke Universiteit Nijmegen, Nederland, 1993.

[14] H. van Imhoff. Riesz* homomorphisms on pre-Riesz spaces consisting of continuous functions. Positivity, 22(2):425-447, 2018.

[15] J. van Waaij. Tensor products in Riesz space theory. Master's thesis, Mathematisch Instituut, Universiteit Leiden, Nederland, 2013. 